\title{
Motivación y TIC como reguladores de la actividad física en adolescentes: una revisión sistemática \\ Motivation and ICT as regulators of physical activity in teenagers: a systematic review
}

\author{
Miguel Ángel Durán-Vinagre, Víctor Manuel Leador Albano, Susana Sánchez Herrera, Sebastián Feu \\ Universidad de Extremadura (España)
}

\begin{abstract}
Resumen. Tradicionalmente, la diversidad de factores que influyen en la práctica de actividad física de los adolescentes ha sido ampliamente estudiada. Sin embargo, no hemos localizado investigaciones que aporten datos empíricos que permitan determinar el efecto de la motivación y el uso de las TIC en la práctica de actividad física en adolescentes. Por ello, el objetivo principal de este estudio fue realizar una revisión sistemática de los estudios que evalúan el efecto de la motivación y el uso de las TIC para la mejora de la práctica de actividad física en la adolescencia. En la revisión fueron incluidos 243 artículos científicos publicados en las bases de datos Web of Science (WOS) y Scopus, desde enero de 2010 hasta abril de 2021, utilizando la metodología PRISMA para analizar la información. Los resultados arrojan evidencias científicas que justifican que la motivación y el uso de las TIC son factores que inciden positivamente en la realización de actividad física. Entre las principales razones destaca que el uso inapropiado de los recursos tecnológicos es uno de los principales causantes del sedentarismo, la inactividad física y la declinación de los hábitos de vida saludables de la población.
\end{abstract}

Palabras clave: motivación, TIC, actividad física, adolescentes, análisis bibliométrico.

\begin{abstract}
Traditionally, the diversity of factors that influence the practice of physical activity of adolescents has been widely studied. However, we have not found research that provides empirical data that allow us to determine the effect of motivation and the use of ICT in the practice of physical activity in adolescents. Therefore, the main objective of this study was to carry out a systematic review of the studies that evaluate the effect of motivation and the use of ICT to improve the practice of physical activity in adolescence. The review included 243 scientific articles published in the Web of Science (WOS) and Scopus databases, from January 2010 to April 2021, using the PRISMA methodology to analyze the information. The results provide scientific evidence that justify that motivation and the use of ICT are factors that positively affect the performance of physical activity. Among the main reasons, it stands out that the inappropriate use of technological resources is one of the main causes of sedentary lifestyle, physical inactivity and the decline in healthy lifestyle habits of the population.
\end{abstract}

Keywords: motivation, ICT, physical activity, adolescent, bibliometric analysis.

\section{Introducción}

La adolescencia es un periodo de transición de la niñez a la edad adulta en el que se producen múltiples cambios biológicos, psicológicos y sociales (GüemesHidalgo, Ceñal \& Hidalgo, 2017). Además, constituye una etapa significativa en la configuración de estilos de vida saludables (Holmberg et al., 2018; Kristo, Gültekin, Öztag \& Sikalidis, 2020). En este sentido, la realización de actividad física (en adelante A.F) durante la adolescencia es un indicador clave para establecer prácticas de vida saludable, destacando, por una parte, la disminución de los niveles de sedentarismo e inactividad física en los adolescentes (Kumar, Robinson \& Till, 2015; Menéndez \& González, 2019), y por otra la estimulación

Fecha recepción: 02-03-21. Fecha de aceptación: 27-05-21

Miguel Ángel Durán Vinagre

mduranv@unex.es del desarrollo físico, cognitivo y social (Duda \& Ntoumanis, 2003; Guillamón, Canto \& López, 2019).

En los últimos años, las investigaciones sobre la A.F. y la salud han aumentado considerablemente, encontrándose estudios que demuestran que la práctica regular y continuada de A.F. presenta una relación directa entre la mejora de la salud y el incremento de la esperanza de vida (Barbosa \& Urrea, 2018; Lavie, Ozemek, Carbone, Katzmarzyk \& Blair, 2019). En consonancia, Cruz-Santos, González-Rivera \& Rosario-Rodríguez (2019) analizaron la actitud sobre el ejercicio físico y el deporte con una población de 255 adolescentes, obteniendo una correlación positiva y estadísticamente significativa entre la actitud hacia el ejercicio físico y el deporte, el bienestar psicológico y la satisfacción con la vida. Lavie et al. (2019), en su revisión sistemática de los comportamientos sedentarios, el ejercicio y la salud, constataron que existen beneficios de la A.F. para prevenir enfermedades no transmisibles. Resultados 
similares se muestran en estudios que detallan y evidencian que existen informes y documentos que muestran los múltiples beneficios que ofrece la A.F. en relación con la salud, al igual que los comportamientos saludables ideales (Rippe, 2019; Schinke, McGannon \& Smith, 2016).

A pesar de las evidencias científicas, la inactividad física se ha convertido en uno de los principales problemas de la sociedad actual tanto en el contexto nacional como en el internacional, localizándose estudios que demuestran que son bajos los porcentajes de los adolescentes que realizan A.F (Baqal, Saleheen \& Albuhairan, 2020; Práxedes, Sevil, Moreno, del Villar \& GarcíaGonzález, 2016). Igualmente, diversas las investigaciones que han indagado sobre la prevalencia de la A.F. en adolescentes (Ogdel, Carroll, Lawman, Fryar, KruszonMoran, Kit \& Flegal, 2016; Vidarte, Vélez, Sandoval \& Alfonso, 2011).A este respecto, Al-Nakeeb, Lyons, Dodd \& Al-Nuaim (2015) analizaron el estilo de vida, los hábitos de salud y los factores de riesgo entre los adultos jóvenes, poniendo énfasis en la información sustancial y la prevalencia que manifiesta la A. F. En esta línea, Martínez et al., (2012) o Rico-Díaz, Arce-Fernández, Padrón-Cabo, Peixoto-Pino \& Abelairas-Gómez (2019) trataron de determinar cuáles eran los motivos que producían el descenso progresivo o de abandono de la práctica deportiva de adolescentes e identificaron que uno de los desencadenantes fundamentales era la caída de la motivación hacia la A.F. Otro estudio que pretendía conocer la cantidad de actividades físico-deportivas realizada por los jóvenes obtuvieron que la media de los participantes se situaba por debajo de los tres días a la semana de A.F. (Durán-Vinagre, Sánchez \& Feu, 2019). De estos resultados, se desprende que la población objeto de estudio prioriza otras actividades cotidianas y de ocio sin valorar los beneficios que genera A.F. tanto en el plano físico como mental (Molina-Castillo, Castillo \& Pablos, 2007; Vázquez \& Mesa, 2012).

Por otro lado, y con el fin de dar una explicación a estos datos, la A.F. se ha relacionado con otros factores psicológicos como los procesos cognitivos (Doherty \& Forés, 2019), los procesos emocionales (Ferrer \& Berry, 2018), el clima social (Yun et al., 2018) y principalmente con la motivación (Chacón, Zurita, Castro, Espejo, Martínez \& Pérez 2017; Franco, Coteron \& Gómez, 2017). Este último constructo psicológico juega un papel imprescindible en la práctica de A.F. en la ciudadanía, debido a que el ser humano se comporta en función a una serie de conductas motivacionales a la hora de lograr metas y objetivos en diferentes contextos
(Almagro, Navarro, Paramio \& Sáenz-López, 2015). A este respecto, la motivación determina la dirección y la intensidad de la conducta a la hora de participar en actividades físico-deportivas, entendiéndose por dirección aquellas posibles metas que una persona manifiesta al sentirse atraído por ella y por intensidad a la cantidad de empeño y esfuerzo que una persona emplea para alcanzar determinadas metas (Escartí \& Brustad, 2002). Tanto la intensidad como la dirección que adoptan los motivos de práctica son relevantes para la continuidad en una A.F. y/o deportiva (Cervelló, Escartí \& Guzmán, 2007).

La Teoría de la Autodeterminación (Deci \& Ryan, 1985), constituye un modelo explicativo de la motivación del ser humano que se ha aplicado a diversos ámbitos y contextos, entre ellos en la actividad físico-deportiva (Moreno-Murcia, 2011). Años después, Deci \& Ryan (2012), en la misma línea de sus primeras aportaciones, especificaron que dicha teoría empírica de la motivación humana y de la personalidad distinguían la motivación autónoma y controlada, es decir, aquella motivación autodeterminada o no determinada. De igual modo, esta teoría se presenta dentro de un continuo abordando distintos grados de autodeterminación de la conducta, encontrando diferentes tipos de motivación que se sitúa en un extremo la motivación intrínseca, seguido de la motivación extrínseca y en el otro la desmotivación (Deci \& Ryan, 2000). La primera de ellas se relaciona con las necesidades de exploración del entorno, la curiosidad y el placer generado al realizar una actividad, constituyendo en sí dicha actividad como un objetivo a perseguir y la gratificación correspondiente, suscitando, además, sensaciones de competencia y autorrealización (Moreno \& Martínez, 2006). Sin embargo, la motivación extrínseca está asociado con aquellas conductas no autodeterminadas, que englobarían un continuo que abarca desde la desmotivación, la regulación externa, introyectada, identificada e integrada (García del Castillo, García del Castillo, López-Sánchez \& Dias, 2015; Moreno \& Martínez, 2006).

En la actualidad, el uso de las TIC, y en especial, la utilización de los dispositivos móviles, han ocupado un papel importante en la vida de las personas, adquiriendo gran protagonismo en la gestión del tiempo, el ocio, las relaciones sociales o incluso, el control y desarrollo de la práctica de A.F. (Naslund, Bondre, Torous \& Aschbrenner, 2020). En este sentido, algunos trabajos han analizado el uso de las TIC para motivar la realización de A.F., bien sea mediante feedbacks, juegos, mensajes interactivos, control de variables de carga, sms, 
entre otras (Simons, Chinapaw, Bovenkamp, de Boer, Seidell, Brug \& de Vet, 2014; Tong et al., 2019). Las TIC pueden ser un recurso potenciador de la vida saludable y un factor condicionante de la A.F. (Diego-Cordero, Fernández-García \& Badanta-Romero, 2017; Spanakis et al., 2016), sin embargo, estas herramientas emplean diferentes estrategias motivacionales y recursos para incentivar la práctica de A.F. (Quevedo-Blasco, Quevedo-Blasco \& Bermúdez, 2009).

El uso de las aplicaciones en dispositivos móviles y de las nuevas tecnologías ha contribuido y sigue contribuyendo a un incremento en la evaluación de la salud (Baxter, Carroll, Keogh \& Vandalanotte, 2020; Fanning, Mullun \& McAuley, 2012; García, Jimmefors, Mausavi, Adrianson, Rosenberg \& Archer, 2015), encontrándose recursos que permiten combinar los objetivos de salud con un sistema de seguimiento (Schoeppe, Alley, Van Lippevelde, Bray, Williams, Duncan \& Vandelanotte, 2016). En consonancia y más específicamente, las aplicaciones móviles o «apps» se han erigido como un recurso mediante las cuales se pueden llegar a cambiar las percepciones de los adolescentes, canalizando su motivación, interés y disfrute hacia la realización de actividad física de forma autónoma, voluntaria y prolongada en el tiempo (González, Sánchez-Oliva, Sánchez-Miguel, González-Ponce \& García-Calvo, 2016).

Este trabajo pretende, no sólo dar visibilidad a los hallazgos documentados sobre el uso de las TIC para motivar la práctica de A.F., sino también estudiar cómo se ha abordado metodológicamente la investigación en este campo y los resultados obtenidos en esta línea de investigación. Asimismo, esta revisión sistemática indaga la relación existente entre las variables objeto de estudio y permite extraer las fortalezas y debilidades de estos trabajos con el fin de potenciar las futuras líneas de investigación. Por este motivo, se realizó una revisión sistemática de la literatura de estudios empíricos por los siguientes objetivos: (i) cono- Tabla 1.

cer el estado de la investigación sobre las TIC como herramienta de la motivación de la A.F. (ii) identificar y analizar las investigaciones que estudian la asociación de la motivación y el uso de las TIC como factores que influyen en la realización de A.F. en la adolescencia.

\section{Materiales y método}

\section{Diseño del estudio}

El estudio realizado es una revisión sistemática de la literatura especializada cuyo tema de estudio es la motivación y el uso de las TIC para la mejora de la práctica de A.F. en adolescentes. El diseño de la investigación es de tipo teórico (Ato, López \& Benavente, 2013). Los datos fueron recogidos a través de un desarrollo sistemático de la metodología para la selección de los artículos, la definición de las variables, la codificación y el análisis de los datos. Se utilizó la metodología PRISMA para el desarrollo de la investigación (Preferred Reporting Items for Systematic reviews and Meta-Analyses) (Moher et al., 2010, 2015), formada por cuatro fases: (a) identificación, (b) selección, (c) elegibilidad e (d) inclusión. Se empleó el protocolo PICO (Landa-Ramírez \& Arredondo-Pantaleón, 2014) para definir el objeto de estudio, buscando asociaciones en los adolescentes para determinar los efectos de la motivación, a través de las TIC, en la práctica de A.F. en comparación con otros tipos de motivación o ausencia de misma.

El periodo de análisis está comprendido entre el año 2010 y abril de 2021. Los adolescentes analizados en este intervalo de tiempo han estado en contacto con las tecnologías desde su infancia, mostrando una familiarización y uso de las TIC en su vida diaria.

\section{Estrategia de búsqueda}

En la determinación de los artículos seleccionados se han consultado las bases de datos, indexados en el área de ciencias sociales o en la de educación de la WOS y Scopus en abril de 2021 utilizando palabras clave, operadores booleanos y opciones de búsqueda avanzada. Para dicha búsqueda se consideraron los siguientes términos y booleanos localizados en el Tesauro de la base de datos Educational Resource Information Center (ERIC) siendo: (Physical Activity) AND (Motivation) AND (Information Technology) OR (Web 2.0 Technologies) AND (Adolescents).

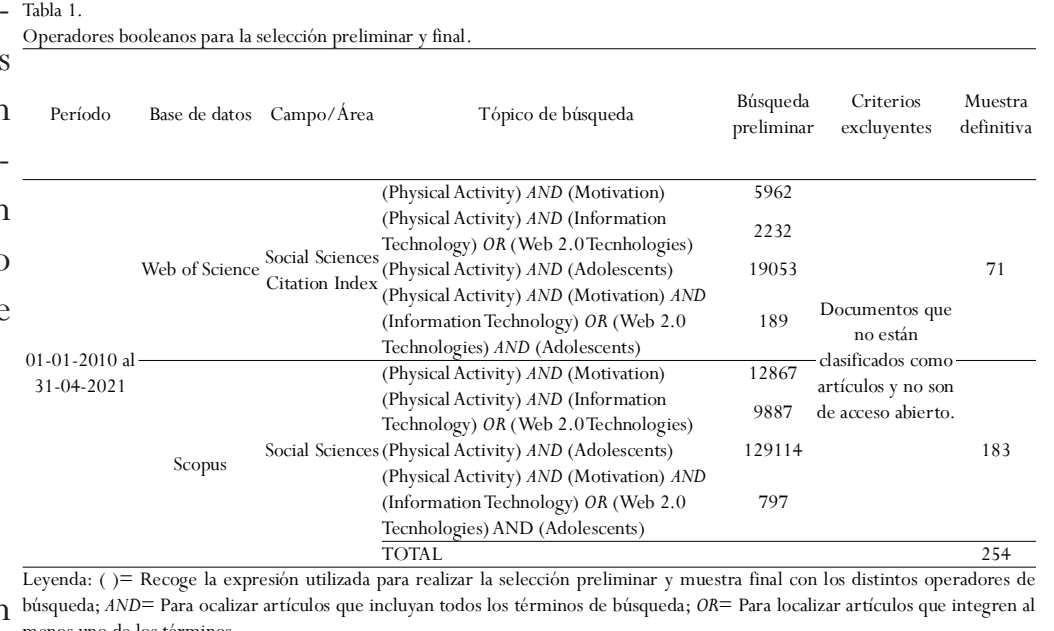
mes uno des términos. 
Para la recogida de información se han manejado con diversos operadores de búsqueda, atendiendo al tópico de estudio (Tabla 1).

Cabe destacar que se eligieron los documentos que pertenecían a los artículos ya que son el tipo de publicación más utilizados y compartidos en el ámbito científico. La mayoría de los trabajos divulgados, además de presentar una estructura y un formato muy similar muestran mayor rigor científico, pues deben ser sometidos al proceso de evaluación y/o de revisión por pares que los equipos editoriales realizan de forma previa a su publicación.

Criterios de inclusión y exclusión

En la muestra final se tuvo en consideración que respondiesen específicamente a los siguientes criterios de inclusión siendo: (1) estudios cuyo objetivo se centra en analizar la motivación y el uso de las TIC para mejorar los hábitos de práctica de A.F.; (2) los participantes son adolescentes, de 15 a 29 años (Krauskopf, 2015); (3) documentos escritos en inglés o español; (4) el documento es un artículo; (5) acceso abierto del documento. Los estudios se excluyeron si: (1) incluáan otros grupos de edad distintos a los adolescentes; (2) inaccesibilidad a la versión de texto completo; (3) no incluían datos relevantes para este estudio.

\section{Extracción de los datos}

El protocolo realizado para la definición de las categorías de los artículos incluyó las siguientes características: paradigma, tipo de estrategia, tipo de motivación, medición de A.F., propuesta de A.F., feedback motivacional y tipo de TIC empleada.

Para el paradigma se plantearon tres opciones, que se asocia a lo cuantitativo, cualitativo y mixto. También se establecieron cuatro tipos de estrategias siguiendo las características descritas por Ato et al. (2013) siendo (1) manipulativa, (2) asociativa y (3) descriptiva.

Respecto al tipo de motivación las investigaciones se clasificaron si respondían a (1) motivación intrínseca o (2) extrínseca. La primera se relaciona con las necesidades de exploración del entorno, la curiosidad y el placer generado al realizar una actividad, constituyendo en sí un objetivo a perseguir y la gratificación correspondiente, suscitando, además, sensaciones de competencia y autorrealización (Moreno \& Martínez, 2006). Sin embargo, la motivación extrínseca está asociado con aquellas conductas no autodeterminadas, que englobarían un continuo que abarca desde la desmotivación, la regulación externa, introyectada, identificada e integrada (García del Castillo et al., 2015; Moreno \& Martínez,
2006).

En cuanto a la catalogación de las investigaciones en base a la medición de A.F. las opciones fueron estudios que se enmarcaran en (1) cuestionarios de hábitos de A.F., (2) pruebas de A.F., (3) abordan situaciones de A.F. considerando el uso de las TIC como recurso mediador y motivador (4) incluía cuestionario y prueba de A.F. Atendiendo al tipo de TIC aplicada, los investigadores se clasificaron si correspondían a (1) app, (2) juegos interactivos, (3) recursos web u (4) otros recursos (vídeos, pulseras, libro electrónico, ...). En el proceso de codificación para el análisis de la calidad del dato participaron dos investigadores con los que se analizó la fiabilidad inter-observador a través del cálculo del Índice Kappa de Cohen.

\section{Calidad de los estudios}

Para evaluar la calidad de los estudios, se utilizaron unos criterios de calidad para analizar las publicaciones compuesto por 16 ítems (Law, Stewart, Pollock, Letts, Bosch \& Westmorland, 1998; Sarmento, Clemente, Araújo, Davids, McRobert \& Figueiredo, 2018). Los documentos fueron evaluados según: propósito (Q1), relevancia de los antecedentes literatura $(\mathrm{Q} 2)$, adecuación del diseño del estudio (Q3), muestra incluida (Q4 y Q5), procedimiento de consentimiento informado (Q6), medidas de resultado (Q7 y Q8), descripción del método (Q9), importancia de los resultados (Q10), análisis (Q11), importancia práctica (Q12), descripción de los abandonos (Q13), conclusiones (Q14), implicaciones prácticas (Q15) y limitaciones (Q16). Todos los criterios de calidad se puntuaron en una escala binaria (0/1), en donde dos de esos criterios (Q6 y Q13) presentaron la opción: «Si no corresponde, suponga N/A (no aplicable)». La puntuación final se expresa en porcentaje, enmarcándose en tres rangos en función de la calidad metodológica: baja (50\%), buena (51-75\%) y excelente (>75\%) (Sarmento et al., 2018).

\section{Análisis estadístico}

Se realizó un análisis descriptivo de todas las variables registradas en la investigación. También se efectuó un análisis de respuesta múltiple sobre las siguientes variables: método de investigación, estrategia asociativa, tipo de motivación, medición de la A.F., propuesta de A.F., feedback motivacional y tipo de TIC empleada. El análisis se completó con tablas y figuras de contingencia para identificar las relaciones entre las variables de investigación (Ibáñez, García-Rubio, Antúnez \& Feu, 2019). En cuanto al análisis cualitativo de los estudios, 
se evaluó el grado de acuerdo entre los observadores para el 100\% de los estudios $(n=17)$ dado por el valor del índice de Kappa de Cohen (Landis \& Kock, 1977).

\section{Resultados}

\section{Selección de los estudios}

La Figura 1 muestra el proceso de selección de los artículos. Éstos se incluyeron siempre que presentaran una serie de requisitos de inclusión. Para dicho proceso se tuvo en cuenta cuatro fases: (a) identificación, (b) selección, (c) elegibilidad e (d) inclusión.

La búsqueda inicial identificó 254 resultados de acceso abierto y clasificados como «artículos». Estos datos se exportaron al software de administrador de referencias (EndNote Online) y se procedió a eliminar aquellos registros duplicados siendo once en este caso. De

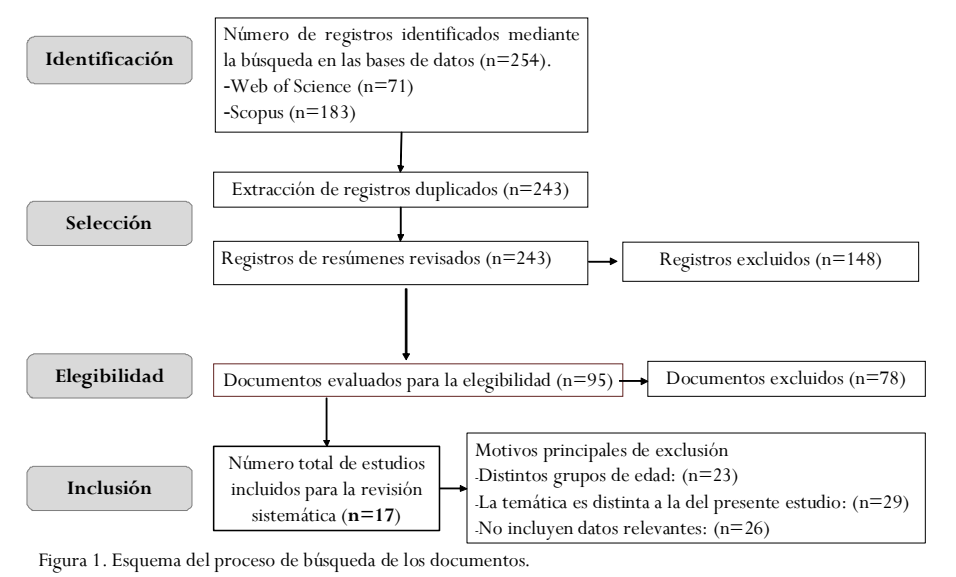

los 243 documentos restantes, 95 de ellos fueron evaluados para la elegibilidad, excluyendo 148. Tras realizar un análisis más profundo, diecisiete estudios cumplieron con los criterios de elegibilidad y se incluyeron en los resultados de esta revisión (Figura 1).

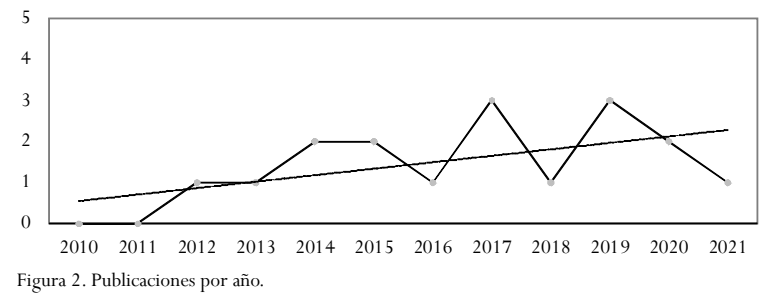

\section{Resultados por año}

Desde el inicio del período de análisis (2010), ha habido un incremento de las publicaciones sobre el tópico de investigación, sobre todo en los últimos años (Figura 2). En cuanto a la frecuencia de artículos por año, en 2017 y 2019 se publicaron tres documentos, seguido de 2014, 2015 y 2020 con dos trabajos durante esos periodos. Existe una progresión lineal en la producción de estos artículos que muestra una tendencia creciente en el período analizado $\left(\mathrm{r}^{2}=.324\right)$, aunque se aprecie una carencia en el tópico investigado tras el resultado arrojado.

\section{Resultados de la tabla de contingencia} de las variables del estudio

En la Tabla 2 se presentan el abordaje metodológico de los estudios seleccionados.

Tabla 2.

\begin{tabular}{|c|c|c|c|c|c|c|c|c|c|c|c|c|c|c|c|c|c|c|}
\hline \multicolumn{3}{|c|}{ Método de Investigación } & \multicolumn{3}{|c|}{ Tipo de motivación } & \multicolumn{4}{|c|}{ Mide AF } & \multicolumn{2}{|c|}{$\begin{array}{c}\text { Propone hacer } \\
\text { AF } \\
\end{array}$} & \multicolumn{2}{|c|}{$\begin{array}{c}\text { Feedback } \\
\text { Motivacional }\end{array}$} & \multicolumn{4}{|c|}{ TIC aplicada } & \multirow[b]{2}{*}{ Total } \\
\hline $\begin{array}{l}\text { Paradigma } \\
\text { Estrategia }\end{array}$ & $\begin{array}{c}\text { Tipo de } \\
\text { estrategia }\end{array}$ & & Intrínseca & Extrínseca & $\begin{array}{c}\text { Ambos } \\
\text { tipos }\end{array}$ & $\begin{array}{l}\text { Cuestionario } \\
\text { hábitos A.F. }\end{array}$ & $\begin{array}{l}\text { Prueba } \\
\text { de A.F. }\end{array}$ & $\begin{array}{l}\text { Aborda A.F. } \\
\text { mediante el } \\
\text { uso de TIC }\end{array}$ & $\begin{array}{c}\text { Incluye } \\
\text { cuestionario } \\
\text { y prueba }\end{array}$ & $\mathrm{Si}$ & No & $\mathrm{Si}$ & No & App & Juegos & Web & Otros & \\
\hline \multirow{6}{*}{ Cuantitativo } & \multirow[b]{2}{*}{ Manipulativa } & $\mathrm{n}$ & 2 & 4 & 1 & 1 & 1 & 4 & 1 & 6 & 1 & 5 & 2 & 2 & 2 & 1 & 2 & 7 \\
\hline & & $\%$ estrategia & $28.6 \%$ & $57.1 \%$ & $14.3 \%$ & $14.3 \%$ & $14.3 \%$ & $57.1 \%$ & $14.3 \%$ & $85.7 \%$ & $14.3 \%$ & $71.4 \%$ & $28.6 \%$ & $28.6 \%$ & $28.6 \%$ & $14.3 \%$ & $28.6 \%$ & $100 \%$ \\
\hline & \multirow[b]{2}{*}{ Asociativa } & $\mathrm{n}$ & 2 & 1 & 0 & 0 & 1 & 2 & 0 & 2 & 1 & 2 & 1 & 2 & 0 & 1 & 0 & 3 \\
\hline & & $\%$ estrategia & $66.6 \%$ & $33.3 \%$ & $0 \%$ & $0 \%$ & $33.3 \%$ & $66.6 \%$ & $0 \%$ & $66.6 \%$ & $33.3 \%$ & $66.6 \%$ & $33.3 \%$ & $66.6 \%$ & $0 \%$ & $33.3 \%$ & $0 \%$ & $100 \%$ \\
\hline & \multirow[b]{2}{*}{ Total } & $\mathrm{n}$ & 4 & 5 & 1 & 1 & 2 & 6 & 1 & 8 & 2 & 7 & 3 & 4 & 2 & 2 & 2 & 10 \\
\hline & & $\%$ estrategia & $40 \%$ & $50 \%$ & $10 \%$ & $10 \%$ & $20 \%$ & $60 \%$ & $10 \%$ & $80 \%$ & $20 \%$ & $70 \%$ & $30 \%$ & $40 \%$ & $20 \%$ & $20 \%$ & $20 \%$ & $100 \%$ \\
\hline \multirow{4}{*}{ Cualitativo } & Descriptiva & $\mathrm{n}$ & 1 & 1 & 0 & 1 & 0 & 1 & 0 & 0 & 2 & 0 & 2 & 0 & 0 & 0 & 2 & 2 \\
\hline & \multirow{3}{*}{ Total } & $\%$ estrategia & $50 \%$ & $50 \%$ & $0 \%$ & $50 \%$ & $0 \%$ & $50 \%$ & $0 \%$ & $0 \%$ & $100 \%$ & $0 \%$ & $100 \%$ & $0 \%$ & $0 \%$ & $0 \%$ & $100 \%$ & $100 \%$ \\
\hline & & $\mathrm{n}$ & 1 & 1 & 0 & 1 & 0 & 1 & 0 & 0 & 2 & 0 & 2 & 0 & 0 & 0 & 2 & 2 \\
\hline & & $\%$ estrategia & $50 \%$ & $50 \%$ & $0 \%$ & $50 \%$ & $0 \%$ & $50 \%$ & $0 \%$ & $0 \%$ & $100 \%$ & $0 \%$ & $100 \%$ & $0 \%$ & $0 \%$ & $0 \%$ & $100 \%$ & $100 \%$ \\
\hline \multirow{6}{*}{ Mixto } & Manipulativa & $\mathrm{n}$ & 2 & 0 & 2 & 1 & 1 & 1 & 1 & 2 & 2 & 1 & 3 & 2 & 0 & 1 & 1 & 4 \\
\hline & \multirow{3}{*}{ Descriptiva } & $\%$ estrategia & $50 \%$ & $0 \%$ & $50 \%$ & $25 \%$ & $25 \%$ & $25 \%$ & $25 \%$ & $50 \%$ & $50 \%$ & $25 \%$ & $75 \%$ & $50 \%$ & $0 \%$ & $25 \%$ & $25 \%$ & $100 \%$ \\
\hline & & $\mathrm{n}$ & 1 & 0 & 0 & 1 & 0 & 0 & 0 & 1 & 0 & 0 & 1 & 0 & 0 & 1 & 0 & 1 \\
\hline & & $\%$ estrategia & $100 \%$ & $0 \%$ & $0 \%$ & $100 \%$ & $0 \%$ & $0 \%$ & $0 \%$ & $100 \%$ & $0 \%$ & $0 \%$ & $100 \%$ & $0 \%$ & $0 \%$ & $100 \%$ & $0 \%$ & $100 \%$ \\
\hline & \multirow[t]{2}{*}{ Total } & $\mathrm{n}$ & 3 & 0 & 2 & 2 & 1 & 1 & 1 & 3 & 2 & 1 & 4 & 2 & 0 & 2 & 1 & 5 \\
\hline & & $\%$ estrategia & $60 \%$ & $0 \%$ & $40 \%$ & $40 \%$ & $20 \%$ & $20 \%$ & $20 \%$ & $60 \%$ & $40 \%$ & $20 \%$ & $80 \%$ & $40 \%$ & $0 \%$ & $40 \%$ & $20 \%$ & $100 \%$ \\
\hline \multirow{8}{*}{ Total } & \multirow[t]{2}{*}{ Manipulativa } & $\mathrm{n}$ & 4 & 4 & 3 & 2 & 2 & 5 & 2 & 8 & 3 & 6 & 5 & 4 & 2 & 2 & 3 & 11 \\
\hline & & $\%$ estrategia & $36.3 \%$ & $36.3 \%$ & $27.2 \%$ & $18.2 \%$ & $18.2 \%$ & $45.5 \%$ & $18.2 \%$ & $72.7 \%$ & $27.3 \%$ & $54.5 \%$ & $45.4 \%$ & $36.4 \%$ & $18.2 \%$ & $18.2 \%$ & $27.3 \%$ & $100 \%$ \\
\hline & \multirow[t]{2}{*}{ Asociativa } & $\mathrm{n}$ & 2 & 1 & 0 & 0 & 1 & 2 & 0 & 2 & 1 & 2 & 1 & 2 & 0 & 1 & 0 & 3 \\
\hline & & $\%$ estrategia & $66.6 \%$ & $33.3 \%$ & $0 \%$ & $0 \%$ & $33.3 \%$ & $66.6 \%$ & $0 \%$ & $66.6 \%$ & $33.3 \%$ & $66.6 \%$ & $33.3 \%$ & $66.6 \%$ & $0 \%$ & $33.3 \%$ & $0 \%$ & $100 \%$ \\
\hline & \multirow[t]{2}{*}{ Descriptiva } & $\mathrm{n}$ & 2 & 1 & 0 & 2 & 0 & 1 & 0 & 1 & 2 & 0 & 3 & 0 & 0 & 1 & 2 & 3 \\
\hline & & $\%$ estrategia & $66.6 \%$ & $33.3 \%$ & $0 \%$ & $66.6 \%$ & $0 \%$ & $33.3 \%$ & $0 \%$ & $33.3 \%$ & $66.6 \%$ & $0 \%$ & $100 \%$ & $0 \%$ & $0 \%$ & $33.3 \%$ & $66.6 \%$ & $100 \%$ \\
\hline & \multirow[t]{2}{*}{ Total } & $\mathrm{n}$ & 8 & 6 & 3 & 4 & 3 & 8 & 2 & 11 & 6 & 8 & 9 & 6 & 2 & 4 & 5 & 17 \\
\hline & & $\%$ estrategia & $47.1 \%$ & $35.3 \%$ & $17.3 \%$ & $23.5 \%$ & $17.6 \%$ & $47.1 \%$ & $11.8 \%$ & $64.7 \%$ & $35.3 \%$ & $47.1 \%$ & $52.9 \%$ & $35.3 \%$ & $11.8 \%$ & $23.5 \%$ & $29.4 \%$ & $100 \%$ \\
\hline
\end{tabular}


Atendiendo al paradigma, los trabajos cuantitativos han sido los más utilizados con un $58.82 \%$, seguido de los paradigmas mixtos con un $29.41 \%$ y los cualitativos con un $11.77 \%$. De las distintas estrategias descritas, la manipulativa muestra un porcentaje más alto en los estudios con un $64.72 \%$ frente al $17.64 \%$ en sendos casos de los estudios de estrategias manipulativa y descriptiva. Se obtuvo que el $76 \%$ se trataban de estudios longitudinales, por lo que el porcentaje restante (24\%) correspondía al tipo transversal.

Prestando atención a los porcentajes arrojados en los resultados de los estudios clasificados cuantitativos el 50\% se asociaron con la motivación extrínseca con cinco trabajos y, sin embargo, en los mixtos fue del 60\% en la motivación intrínseca con tres artículos. Atendiendo a los resultados totales de esta variable, los datos revelan de igual forma que la motivación intrínseca (47.1\%) y extrínseca $(35.3 \%)$ presentan los porcentajes más altos.

Si tenemos en cuenta la medición de la A.F. apreciamos que gran parte de los resultados, concretamente el 44.1\% de los trabajos abordan situaciones de A.F. considerando el uso de las TIC como recurso mediador de dichas situaciones (8), seguido de aquellos documentos que tratan los hábitos de A.F. mediante cuestionarios con un 23.5\% (4), y los que aplican pruebas de A.F. con un $17.6 \%$ (3).

Relacionando el tipo de paradigma y estrategia con los estudios que proponen o no realizar A.F. se aprecia, a tenor de los resultados, que los estudios clasificados como cuantitativos y manipulativos registran seis trabajos y representan el $85.7 \%$ del total (10) que sí lo proponen. De lo contrario, las únicas investigaciones registradas en el tipo cualitativo y descriptivo (2) no proponían hacer A.F.

Nuevamente los estudios dentro de cuantitativo y manipulativo son los que muestran, levemente, una tasa mayor del feedback motivacional con un $71.4 \%$ frente al $28.6 \%$ que no proporciona ningún tipo de retroalimentación. Lo mismo ocurre con los cuantitativos y asociativos donde el $66.6 \%$ es para el sí facilitan ese feedback y lo restante (33.4\%) para los que no. A pesar de ello, si apreciamos el computo de los datos totales, los trabajos que no muestran un feedback a los participantes tienen un resultado más favorable $(52.9 \%)$ respecto a los que sí (47.1\%).

Haciendo alusión al tipo de TIC empleada por las investigaciones analizadas, existe un recuento muy parejo entre las diferentes categorías clasificadas, siendo las aplicaciones (app) las más empleadas (35.3\%). Detallando estos datos en los resultados en la estrategia manipulativa encontramos que tanto las apps como otros recursos (vídeos, pulseras, libro electrónico, ...), los porcentajes más favorables se declinaron sobre estas variables con el $36.36 \%$ y el $27.27 \%$ respectivamente. Por lo contrario, los juegos interactivos y las páginas web representaron el $18.18 \%$ en sendos casos.

Finalmente, hay que apuntar que, de los diecisiete artículos analizados en esta revisión, nueve de ellos mostraban significación en sus resultados (52.94\%), mientras que el resto no incluían o no correspondían la obtención de dicho dato estadístico. Igualmente, de todos los archivos únicamente cuatro $(23.53 \%)$ incluyeron el tamaño del efecto para mostrar la fuerza o magnitud del efecto del fenómeno estudiado.

\section{Calidad de los estudios}

Para analizar la calidad de los estudios seleccionados, se utilizaron los criterios descritos con anterioridad de Law et at. (1998) y Sarmento et al. (2018). El resultado del análisis de confiabilidad entre codificadores se obtuvo calculando el Índice de Kappa arrojando un valor de $.93(p<.05)$, lo que indica una fuerza de concordancia óptima entre los observadores. Los criterios de calidad de los indicadores para los estudios seleccionados fueron la siguiente: el promedio del puntaje de la calidad metodológica fue del $88.46 \%$; un artículo logró la puntuación máxima del 100\%; doce estudios alcanzaron una calificación de $>75 \%$ (excelente calidad metodológica); cuatro artículos obtuvieron un puntaje entre 50\% - 75\% (buena calidad metodológica); y un estudio presentó una puntuación más baja de 50\%.

En cuanto a los ítems, los criterios 5, 6, 7, 10, 12, 14 y 16 obtuvieron algunas discrepancias entre los observadores, aunque se encontraron valores superiores al $70 \%$, mientras que, en los criterios restantes, 1, 2, 3, 4, 8, 9, 11, 13 y 15 obtuvieron un coeficiente de Kappa = 1. Asimismo, los ítems Q1 - "¿el propósito del estudio se indicó correctamente?» y Q2 - "¿se revisó la literatura de fondo relevante?» fueron los únicos que presentaron una puntuación máxima por los observadores. De los artículos seleccionados, un ítem reflejaba principalmente deficiencia metodológica, siendo el Q13 - "ise informó de abandonos?» (25\%). A este le siguen los ítems Q16"¿las limitaciones fueron reconocidas y descritas?" (68.75\%), Q7 - «ifueron confiables las medidas de resultados?» (75\%) y Q10 - «ise informaron los resultados en términos de significación estadística?» (75\%). 
Tabla 3.

Caracteristicas de los estudios analizados.

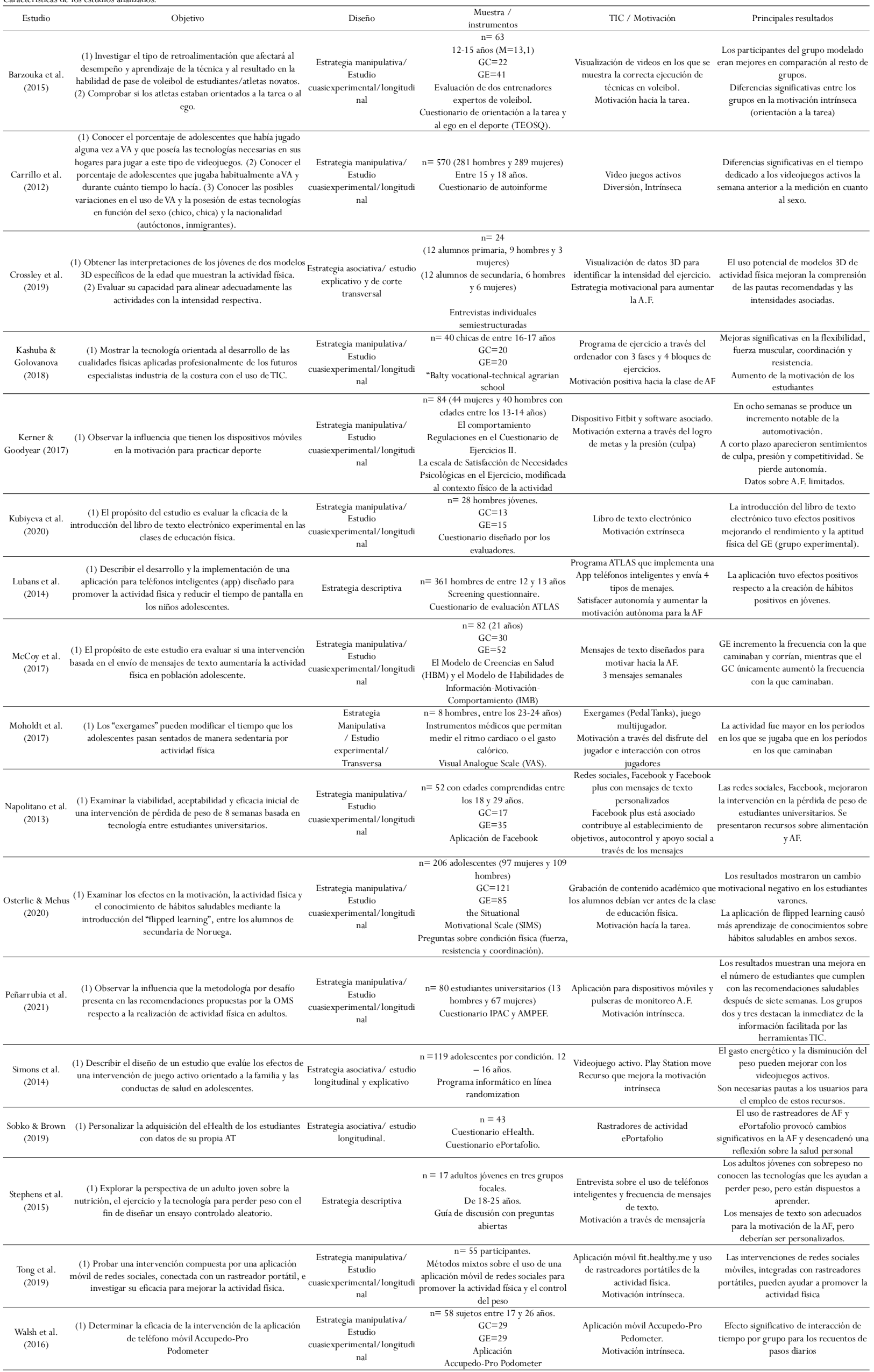




\section{Discusión}

El objetivo de esta investigación fue realizar una revisión sistemática de la literatura de estudios empíricos que evalúan el efecto de la motivación y el uso de las TIC para la mejora de la práctica de A.F. en la adolescencia a partir de las bases de datos Web Of Science (WOS) y Scopus en un periodo comprendido entre enero de 2010 y abril de 2021.

Los principales resultados muestran que, de la búsqueda total de documentos con los descriptores seleccionados (243), tan solo el 6.99\% de los artículos cumplen con los criterios de inclusión y versan sobre el tema, y además cuando analizamos la asociación entre las variables que giran en torno al objetivo del trabajo, únicamente 17 trabajos lo hacen. Sin embargo, algunas investigaciones han documentado que la motivación y el uso de las TIC es uno de los factores más importantes para aumentar la A.F. (Parra, Gómez \& Pintor, 2014; Rhodes, Janssen, Bredin, Warburton \& Bauman, 2017).

En relación con el año de publicación, se ha observado, a través del análisis de progresión línea, que la tendencia en ambas bases de datos es creciente, dejando entrever, en cierta medida, la importancia que la sociedad está otorgando a los factores que influyen en la A.F. de los adolescentes (Howie, Daniels \& Guagliano, 2020). Los mecanismos o recursos que han relacionado la A.F. y el uso de las TIC como elemento motivador de su práctica, permiten determinar que existe un fomento y un apoyo consistente en su aplicación y que, en definitiva, están proyectado la mejora y la participación activa de los sujetos que intervienen en su puesta en práctica ofreciendo diferentes estrategias para ello (Crossley, McNarry, Rosenberg, Knowles, Eslambolchilar \& Mackintosh, 2019; Lubans, Smith, Skinner \& Morgan, 2014; Tong et al., 2019). Por tanto, cada vez se sigue investigando más sobre ello para tratar de responder a las distintas incógnitas y factores que se asocian a dicha población. Esta tendencia creciente puede explicarse si se tiene en cuenta el interés existente en el ámbito científico por el conocimiento de los fenómenos o efectos que influyen a la hora de practicar A.F. y que, sin lugar a duda, están relacionados con los procesos sociales y psicológicos (Carbonell, Antoñanzas \& Lope, 2018).

La motivación intrínseca fue la más utilizada en los estudios analizados, empleando la diversión propia de los juegos (Carrillo, Carrillo, Murcia, Gimeno \& Carretero, 2012) y la orientación a la tarea (Barzouka, Sotiropoulus \& Kioumoutzoglou, 2015). Así pues, he- mos podido extraer de los trabajos analizados que, algunos de ellos, utilizan mensajes de texto para reforzar e incentivar los progresos del sujeto y generar un estado motivacional más activo y dirigido a la realización de A.F. En este sentido, Moreno \& Martínez (2006) aseguran que este tipo de motivación es necesaria para conocer las necesidades fundamentales de competencia y autonomía en los individuos, pues es una forma de incrementar la satisfacción del bienestar personal (Carrón, Muñoz, Román, Batista \& Castuera, 2016; Deci \& Ryan, 2008). En esta línea, Moreno, Hernández \& GonzálezCutre (2009) consideran que aquellas conductas que se relacionan con la motivación intrínseca se sustentan por la satisfacción, el placer y la diversión que genera el realizar actividades físico-deportivas, de ahí su alto valor predictivo (Méndez-Giménez, Cecchini, FernándezRio \& González, 2012). A pesar de ello, si bien es cierto que los niveles de motivación extrínseca vienen incrementándose en las últimas décadas, aún siguen siendo residuales entre los jóvenes (Moreno, Cervelló \& González-Cutre, 2006).

Los resultados obtenidos apuntan que las apps y los recursos web son los más utilizados en la práctica de A.F., pues permiten la monitorización de la A.F. (Moholdt, Weie, Chorianopoulos, Wang \& Hagen, 2017; Peñarrubia-Lozano, Romero-Roso, Olóriz-Nivela \& Lizalde-Gil, 2021; Tong et al., 2019) y además actúan como recurso motivacional de la propia práctica (Kashuba \& Golovanova, 2018; Walsh, Corbett, Hogan, Duggan \& McNamara, 2016). En este sentido, existen evidencias de que el uso de las TIC tiene influencia sobre la actividad física y puede ayudar a las personas a alcanzar sus propias metas (Rivera-Tapia, CedilloRamírez, Pérez-Nava, Flores-Chico \& Aguilar-Enriquez, 2018; Sullivan \& Lachman, 2017). Igualmente, el empleo positivo de estos medios genera un aumento en la motivación de los participantes, facilitando su desarrollo en actividades de forma más interactiva (Basterra \& Menescardi, 2020; Vázquez, 2021). A este respecto, los nuevos recursos electrónicos se están diseñando para proporcionar un acceso interactivo entre diferentes dispositivos, programas y aplicaciones que permiten al usuario conocer sus propios niveles de A.F. en diferentes contextos (Fernández, Rueda, Cerero \& Tadeu, 2021; Hannan, Harders, Hing, Climstein, Coombes \& Furness, 2019), generándose una motivación a través de la retroalimentación inmediata que recibe el usuario (Bice, Warren \& McClaran, 2015; McCoy, Leggett, Bhuiyan, Brown, Frye \& Williams, 2017; Mendis et al., 2014). Este feedback procede de la sincronización de datos entre 
programas de software con teléfonos inteligentes que permiten que la evaluación de la A.F. se realice a través de dispositivos móviles (Fanning et al., 2012) o a partir de recursos web (Acosta-Medina, Torres-Barrero, PabaMedina \& Melgarejo-Álvarez, 2020).

Gran parte de los artículos analizados se correspondían con investigaciones dentro del paradigma cuantitativo y de estrategia manipulativa y es que este tipo de estudios arroja mayor validez y fiabilidad de los datos e igualmente permite un mayor control de las variables extrañas (Ato et al., 2013). Los estudios longitudinales fueron los más empleados, este tipo de estrategia es más eficaz por identificar relaciones entre todas las dimensiones evolutivas del sujeto, aportando información entre las semejanzas y las diferencias entre los participantes u obteniendo información respecto a los cambios que experimentan a lo largo del ciclo vital (Delgado \& Llorca, 2004).

Partiendo de la clasificación de las estrategias de investigación de Ato et al. (2013), once estudios se afrontaron desde una estrategia manipulativa mientras que la asociativa y descriptiva sólo fueron tres en ambos casos. Dentro del total de documentos seleccionados, el $64.72 \%$ de los artículos se asoció con la estrategia manipulativa. Esto demuestra la gran importancia e influencia que tiene este tipo de metodología en este ámbito. Cabe destacar que la gran mayoría de archivos fueron clasificados como estudios cuasiexperimentales, mientras que solo un estudio fue de corte experimental. A su vez, priman las investigaciones longitudinales del tipo pretest-postest. Por otro lado, aproximadamente la mitad de los estudios utilizan diferentes tipos de TIC como: apps (Lubans et al., 2014; Tong et al., 2017), uso de redes sociales o mensajería electrónica (Napolitano, Hayes, Bennett, Ives \& Foster, 2013) o videojuegos activos (Moholdt et al., 2017; Simons et al., 2014) con el objetivo de promover o incentivar la actividad física. En cuanto al feedfack motivacional, solo un 47\% de los documentos presentaron algún tipo de retroalimentación durante el proceso de investigaciones. Principalmente consistieron en la recepción de mensajes de texto (McCoy et al., 2017), alcanzar algún tipo de meta o logro (Moholdt et al., 2017) y feedback grupal (Napolitano et al., 2013)

Al hilo de lo expuesto anteriormente, es conveniente apuntar que encontramos trabajos que tratan de justificar y comprender el uso inapropiado de los recursos tecnológicos, cuando desencadenan, sobre todo, conductas sedentarias o incluso acciones antisociales especialmente en niños y adolescentes (Latorre-Román, Bueno-Cruz,
Martínez-Redondo \& Salas-Sánchez, 2020; Napolitano et al., 2013; Panahi \& Tremblay, 2018) por lo que se hace necesario prestar atención a estos factores para utilizar las TIC desde un enfoque que permita cambios significativos en la persona y sean favorables para su desarrollo e implementación (Sobko \& Brown, 2019).

En cuanto a las limitaciones de este estudio, la más significativa fue que se encontraron dificultades para acceder a algunos artículos no publicados en acceso abierto. Algunos trabajos no describen claramente el tipo de motivación empleada y fue necesario analizar en función de la literatura y la codificación de las variables definidas para este estudio.

\section{Conclusiones}

Las TICs se han empleado como recurso para mejorar la motivación hacia la práctica de actividad física. Se ha observado que no predomina ningún tipo de motivación sobre otra, aunque la motivación intrínseca fue la más utilizada. Este tipo de recursos han demostrado ser un medio adecuado para incrementar la motivación y la cantidad de actividad física. Además, en algunos estudios se ha podido determinar que mejoraba la condición física de los usuarios. Por ello, los nuevos recursos electrónicos se están diseñando para proporcionar un acceso interactivo entre diferentes dispositivos y programas que permiten al usuario conocer su nivel de práctica en diferentes contextos e interactuar con otras personas.

Son necesarios estudios que analicen en los adolescentes estos factores y variables, ya que es una etapa en la que se configuran y presentan cambios importantes. Es necesario realizar investigaciones que asocien el uso de recursos tecnológicos y el tipo feedback arrojado a través de los mensajes motivadores hacia la práctica y/ o las recompensas que ofrecen las apps y sus beneficios sobre la realización de A.F. Esta actividad debería ser medida de la forma más objetiva posible valorando sus efectos sobre la condición física.

Las tecnologías pueden ayudar al profesorado a potenciar la práctica de actividad física fuera del contexto formal del aula, favoreciendo la autonomía de los jóvenes para controlar su actividad y participar en nuevos entornos de práctica físico-deportiva. Los docentes adquieren un rol importante en las distintas etapas educativas para enseñar y promover las ventajas de las tecnologías en el fomento y control de la actividad física como mediadores para la mejora de hábitos y estilos de vida más saludables. 


\section{Agradecimientos}

Los autores agradecen la colaboración del Plan Propio de Iniciación a la Investigación, Desarrollo Tecnológico e Innovación de la Universidad de Extremadura 2019, al Grupo de Investigación PSIQUEX y a las ayudas a grupos de la Junta de Extremadura (GR18132) y FEDER, una manera de hacer Europa.

\section{Referencias}

Acosta-Medina, J. K., Torres-Barreto, M. L., Paba-Medina, M. C., \& Melgarejo-Alvarez, M. (2020). Análisis de la gamificación en relación a sus elementos. Universidad Industrial de Santander. Preprint. Hal.

Almagro, B. J., Navarro, I., Paramio, G., \& Sáenz-López, P. (2015). Consecuencias de la motivación en las clases de Educación Física. Revista Digital de Educación Física, 6(34), 26-41.

Al-Nakeeb,Y., Lyons, M., Dodd, L. J., \& Al-Nuaim, A. (2015). An investigation into the lifestyle, health habits and risk factors of young adults. Int.J. Environ. Res. Public Health, 12, 4380-4394. https://doi.org/10.3390/ ijerph120404380

Ato, M., López, J. J., \& Benavente, A. (2013). Un sistema de clasifación de los diseños de investigación en psicología. Anales de Psicología, 29(3), 1038-1059. https://doi.org/ 10.6018/analesps.29.3.178511

Baqal, O. J., Saleheen, H., \& Albuhairan, F. S. (2020). Urgent need for adolescent physical activity policies and promotion: lessons from «Jeeluna». Int.J.Environ. Res. Public Health, 17(12), 4464. https://doi.org/10.3390/ ijerph17124464

Barbosa, S. H., \& Arrea, A. M. (2018). Influencia del deporte y la actividad física en el estado de salud físico y mental: una revisión bibliográfica. Revista Katharsis, 25, 155-173. https: / / doi.org/10.25057/issn.2500-5731

Barzouka, K., Sotiropoulos, K., \& Kioumourtzoglou, E. (2015). The effect of feedback through an expert model observation on performance and learning the pass skill in volleyball and motivation. Journal of Physical Education and Sport, 15(3), 407-416. https://doi.org/10.7752/ jpes.2015.03061

Basterra, J., \& Menescardi, C. (2020). Propuesta de innovación interdisciplinar de contenidos de física en las clases de educación física mediante aplicaciones móviles. $R E$ TOS: Nuevas tendencias en educación física, deporte y recreación, 38, 255-261. https://doi.org/10.47197/ retos.v38i38.73794

Baxter, C., Carroll, J. A., Keogh, B., \&Vandelanotte, C. (2020). Assessment of mobile health apps using built-in smartphone sensors for diagnosis and treatment: systematic survey of apps listed in international curated health app libraries. JMIR Mhealth Uhealth, 8(2), e16741. https: / /doi.org/10.2196/16741

Bice, M., Warren, J., \& McClaran, S. R. (2015). Technology and physical activity motivation. International Journal of Sport and Exercise Psychology, 14, 295-304. https://doi.org/ 10.1080/1612197x.2015.1025811

Carbonell,T., Antoñanzas, J. L., \& Lope, A. (2018). La educación física y las relaciones sociales en Educación Primaria. International Journal of Developmental and Educational Psychology, 2(1). https://doi.org/10.17060/ ijodaep.2018.n1.v2.1225

Carrillo,V. J. B., Carrillo, J. I. B., Murcia, J.A. M., Gimeno, E. C., \& Carretero, C. M. (2012). The use of active videogames among adolescents. Cultura, Ciencia y Deporte, 7(19), 19-24. https://doi.org/10.12800/ccd.v7i19.20

Carrón, M. I. A., Muñoz, S. L., Román, M. L., Batista, M., \& Castuera, R. J. (2016). Predicción de la motivación en las etapas de cambio de ejercicio más activos. RETOS: Nuevas tendencias en educación física, deporte y recreación, 30, 8791. https: / /doi.org/10.47197/retos.v0i30.40619

Cervelló, E., Escartí, A., \& Guzmán, J. F. (2007). Youth sport dropout from the achievement goal Theory. Psicothema, 65-71.

Chacón, R., Zurita, F., Castro, M., Espejo,T., Martínez,A., \& Pérez, A. J. (2017). Motivational climate in sport and its relationship with digital sedentary leisure habits in university students. Saúde e Sociedade, 26(1), 29-39. https: / /doi.org/10.1590/s0104-12902017166561

Crossley, S. G. M., McNarry, M.A., Rosenberg, M., Knowles, Z. R., Eslambolchilar, P., \& Mackintosh, K. A. (2019). Understanding youths' ability to interpret 3D-printed physical activity data and identify associated intensity levels: Mixed-methods study. Journal of Medical Internet Research, 21(2). https://doi.org/10.2196/11253

Cruz-Santos,A., González-Rivera, J.A., \& Rosario-Rodríguez, A. (2019).Attitudes towards physical exercise and sports: A psychometric study in college students. Revista Evaluar, 19(1), 58-72. https://doi.org/10.35670/16674545.v19.n2.25083

Deci, E. L., \& Ryan, R. M. (1985). The general causality orientations scale: Selfdetermination in personality. Journal of Research in Personality, 19, 109- 134.

Deci, E. L., \& Ryan, R. M. (2000). The «what» and «why» of goal pursuits: Human needs and the self-determination of behaviour. Psychological Inquiry, 11, 227-268.

Deci, E. L., \& Ryan, R. M. (2008). Self-determination theory: A macrotheory of human motivation, development and health. Canadian Psychology, 49(3), 182-185. https: / / doi.org/10.1037/a0012801

Deci, E. L., \& Ryan, R. M. (2012). Self-determination theory. En A. W. Kruglanski, P.A. M. Van Lange y E. T. Higgins (Eds.), Handbook of theories social psychology, 1, 416-437. London: SAGE.

Delgado, M., \& Llorca, J. (2004). Estudios longitudinales: 
concepto y particularidades. Revista Española de Salud Pública, 78(2), 141-148.

Diego-Cordero, R., Fernández-García, E., \& Badanta-Romero, B. (2017). Uso de lasTIC para fomentar estilos de vida saludables en niños/as y adolescentes: el caso del sobrepeso. Revista española de comunicación en salud, 8(1), 79-91. https: / / doi.org/10.20318/recs.2017.3607

Doherty,A., \& Forés, A. (2019). Physical activity and cognition: inseparable in the classroom. Frontiers in Education, 4, 1-7. https: / /doi.org/10.3389/feduc.2019.00105

Duda, J. L., \& Ntoumanis, N. (2003). Correlates of achievement goal orientations in physical education. International Journal of Educational Research, 39, 415-436. https: / / doi.org/10.1016/j.ijer.2004.06.007

Durán-Vinagre, M., Sánchez, S., \& Feu, S. (2019). Influencia de la actividad físico - deportiva y la intención de ser físicamente activos en universitarios de la facultad de educación de la UEX. International Journal of Developmental and Educational Psychology, 5(5), 83-96. https: / / doi.org/ 10.17060/ijodaep.2019.n1.v5.1563

Escartí, A., \& Brustad, R. (2002). Estudio de la motivación deportiva desde la perspectiva de la teoría de metas. En: Dosil J, editor. Psicología y rendimiento deportivo. Ourense: GERSAM; 2002. pp. 57-70.

Fanning, J., Mullun, S. P., \& McAuley, E. (2012). Increasing physical activity with mobile devices: A metaanalysis. Journal of Medical Internet Research, 14(6), 61-71. https: / / doi.org/10.2196/jmir.2171

Fernández, J. M., Rueda, M. M., Cerero, J. F., \& Tadeu, P. (2021). Impact of ICT on students with disability in the field physical education: A systematic review. RETOS: Nuevas tendencias en educación física, deporte y recreación, 39, 849-856. retos.v0i39.78602

Ferrer, R., \& Berry, W. (2018). Emotion, health decision making, and health behavior. Psychology and Health, 33(1), 1-16. https://doi.org/10.1080/ 08870446.2017.1385787

Franco, E., Coteron, J., \& Gómez, V. (2017). Relación entre motivación, actividad física realizada en el tiempo libre y la intención futura de práctica de actividad. Estudio comparativo entre adolescentes argentinos y españoles. Revista Euroamericana de Ciencias del Deporte, 6(1), 25-34. https: / /doi.org/10.6018/280371

García del Castillo, J. A., García del Castillo-López, A., LópezSánchez, C., \& Dias, P. (2015). Configuración teórica de la motivación de salud desde la teoría de la autodeterminación. Health and Addictions, 15(2), 151-159. https: / / doi.org/10.21134/haaj.v15i2.252

García, D., Jimmefors, A., Mausavi, F., Adrianson, L., Rosenberg, P., \& Archer, T. (2015). Self-regulatory mode (locomotion and assessment), well-being (subjective and psychological), and exercise behavior (frequency and intensity) in relation to high school pupils academic achievement. PeerJ, 3, e847. https://doi.org/10.7717/ peerj. 847

González, J. J. P., Sánchez-Oliva, D., Sánchez-Miguel, P. A., González-Ponce, I., \& García-Calvo, T. (2016). Proyecto MÓVIL-ÍZATE: Fomento de la actividad física en escolares mediante las Apps móviles. RETOS: Nuevas tendencias en educación física, deporte y recreación, 30, 3-8. https: / / doi.org/10.47197/retos.v0i30.34258

Güemes-Hidalgo, M., Ceñal, M. J., \& Hidalgo, M. I. (2017). Pubertad y adolescencia. Revista de Formación Continuada de la Sociedad Española de Medicina de la Adolescencia, 5(5), 7-22.

Guillamón, A. R., Canto, E. G., \& López, P. J. C. (2019). Actividad física, condición física y autoconcepto en escolares de 8 a 12 años. RETOS: Nuevas tendencias en educación física, deporte y recreación, 35, 236-241. https://doi.org/ 10.14198/jhse.2019.143.03

Hannan, A., Harders, M. P., Hing, W., Climstein, M.,. Coombes, J. S., \& Furness, J. (2019). Impact of wearable physical activity monitoring devices with exercise prescription or advice in the maintenance phase of cardiac rehabilitation: systematic review and meta-analysis. BMC Sports Science, Medicine and Rehabilitation, 11(14), 1-21. https: / / doi.org/10.1186/s13102-019-0126-8

Holmberg, C., Larsson, C., Korp, P., Lindgren, E. C., Jonsson, L., Fröberg, A., ... \& Berg, C. (2018). Empowering aspects for healthy food and physical activity habits: adolescents' experiences of a school-based intervention in a disadvantaged urban community. Internacional Journal of Qualitative Studies on Health andWell-being, 13(1). https: / /doi.org/10.1080/17482631.2018.1487759

Howie, E. K., Daniels, B.T., \& Guagliano, J. (2020). Promoting physical activity through youth sports programs: it's social. American Journal of Lifestyle Medicine, 14(1), 78-88. https: / / doi.org/10.1177/1559827618754842

Ibáñez, S. J., García-Rubio, J., Antúnez, A., \& Feu, S. (2019). Coaching in Spain Research on the Sport Coach in Spain: A Systematic Review of DoctoralTheses. International Sport Coaching Journal, 6(1), 110-125. https://doi.org/ 10.1123/iscj.2018-0096

Kashuba, V. O., \& Golovanova, N. L. (2018). Increase in efficiency of professionally applied physical training of pupils of 16-17 years old based on application of informational and methodical systems. Physical Education of Students, 22(2), 57. https://doi.org/10.15561/ 20755279.2018.0201

Krauskopf, D. (2015). Los marcadores de juventud: La complejidad de las edades. Última década, 23(42), 115-128. https://doi.org/10.4067/s 0718 22362015000100006

Kristo,A. S., Gültekin, B., Öztag, M., \& Sikalidis,A. K. (2020). The effect of eating habits' quality on scholastic performance in turkish adolescents. Behavioral Sciences, 10(1). https: / / doi.org/10.3390/bs10010031 
Kumar, B., Robinson, R., \& Simon,T. (2015). Physical activity and heath in adolescence. Clinical Medicine, 15(3), 267272. https://doi.org/10.7861/clinmedicine.15-3-267

Landa-Ramírez, E., \& Arredondo-Pantaleón, A. J. (2014). Herramienta PICO para la formulación y búsqueda de preguntas clínicamente relevantes en la psiconcología basada en la evidencia. Psicooncología, 11(2-3), 259-270. https: / /doi.org/10.5209/rev_PSIC.2014.v11.n2-3.47387

Landis, J. R., \& Koch, G. G. (1977). The measurement of observer agreement for categorical data. Biometrics, 33, 159-174.

Latorre-Román, P. A., Bueno-Cruz, M. T., Martínez-Redondo, M., \& Salas-Sánchez, J. (2020). Conductas prosociales y antisociales en el deporte escolar. Apunts. Educación Física y deportes, 1(139), 10-18. https://doi.org/10.5672/ apunts.2014-0983.es.(2020/1).139.02

Lavie, C. J., Ozemek, C., Carbone, S., Katzmarzyk, P.T., \& Blair, S. N. (2019). Sedentary behavior, exercise, and cardiovascular health. Circulation Research, 124(5). https:/ /doi.org/10.1161/CIRCRESAHA.118.312669

Law, M., Stewart, D., Pollock, N., Letts, L., Bosch, J., \& Westmorland, M. (1998). Guidelines for critical review formQuantitative studies. Hamilt. Macmaster University.

Lubans, D. R., Smith, J. J., Skinner, G., \& Morgan, P. J. (2014). Development and implementation of a smartphone application to promote physical activity and reduce screentime in adolescent boys. Frontiers in Public Health, 2. https: / /doi.org/10.3389/fpubh.2014.00

Martínez, A. C., Chillón, P., Martín-Matillas, M., Pérez, I., Castillo, R., ... Delgado-Fernández, M. (2012). Motivos de abandono y no práctica de actividad físico-deportiva en adolescentes españoles: estudio Avena. Cuadernos de Psicología del Deporte, 12(1), 45-54.

McCoy, P., Leggett, S., Bhuiyan, A., Brown, D., Frye, P., \& Williams, B. (2017). Text messaging: an intervation to increase physical activity among african american participants in a faith-based, competitive weight loss program. International Journal of Environmental Research and Public Health, 29(14), 326. https://doi.org/10.3390/ ijerph14040326

Méndez-Giménez, A., Cecchini, J. A., Fernández-Rio, J., \& González, C. (2012). Autodeterminación y metas sociales: un modelo estructural para comprender la intención de práctica, el esfuerzo y el aburrimiento en Educación Física. Aula Abierta, 40(1), 51-62.

Mendis, K., Forster, T., Paxton, K., Hyland, K., Yelverton, J., McLean, R., ... \& Steinbeck, K. (2014). Large and forgetten in rural Australia: assessment, attitudes and possible approaches to losing weight in young adult males. BMC Public Health, 14, 243.

Menéndez, D., \& González, C. (2019). Relaciones entre la práctica de actividad física y deportiva, el autoconcepto, la imagen corporal y los hábitos alimentarios en estudiantes de primaria. E-balonmanocom: Revista de Ciencias del Depor- te, 15(1), 79-96.

Moher, D., Liberati, A., Tetzla, J., \& Altman, D. G. (2010). Preferred reporting items for systematic reviews and meta-analyses:The PRISMA statement. Int.J. Surg, 8, 336341. https: / / doi.org/10.1016/j.ijsu.2010.02.007

Moher, D., Shamseer, L., Clarke, M., Ghersi, D., Liberati, A., Petticrew,M., ... \& Group, P.P.(2015). Preferred reporting items for systematic review and meta-analysis protocols (PRISMA-P) 2015 statement. Systematic Reviews, 4(1), 1 9. https://doi.org/10.1186/2046-4053-4-1

Moholdt,T.,Weie, S., Chorianopoulos, K., Wang, A. I., \& Hagen, K. (2017). Exergaming can be an innovative way of enjoyable high-intensity interval training. BMJ Open Sport and Exercise Medicine, 3(1). https://doi.org/10.1136/ bmjsem-2017-000258

Molina-Castillo, J., Castillo, I., \& Pablos, C. (2007). Bienestar psicológico y práctica deportiva en universitarios. Motricidad European Journal of Human Movement, 18, 79. 91.

Moreno, J.A., \& Martínez, A. (2006). Importancia de la Teoría de la Autodeterminación en la práctica físico-deportiva: Fundamentos e implicaciones prácticas. Cuadernos de $P_{s i-}$ cología del Deporte, 6(2), 39-54.

Moreno, J. A., Cervelló, E., \& González Cutre, D. (2006). Motivación autodeterminada y flujo disposicional en el deporte. Anales de Psicología, 22(2), 310-317.

Moreno, J.A., Hernández, A., \& González-Cutre, D. (2009). Complementando la teoría de la autodeterminación con las metas sociales: un estudio sobre la diversión en educación física. Revista Mexicana de Psicología, 26(2), 213-222.

Moreno-Murcia, J.A. (2011). Teoría de la autodeterminación y adherencia al ejercicio físico. Revista Internacional de Ciencias del Deporte, 7(25), 248-249.

Napolitano, M. A., Hayes, S., Bennett, G. G., Ives, A. K., \& Foster, G. D. (2013). Using facebook and text messaging to deliver a weight loss program to college students. Obesity, 21(1), 25-31. https://doi.org/ 10.1038 / oby. 2012.107

Naslund, J. A., Bondre, A., Torous, J., \& Aschbrenner, K. A. (2020). Social Media and Mental Health: benefits, risks, and opportunities for research and practice. Journal of Technology in Behavioral Science, 5, 245-257. https: / / doi.org/10.1007/s41347-020-00134-x

Ogden, C. L., Carroll, M. D., Lawman, H.G., Fryar, C.D., Kruszon-Moran, D., Kit, B.K., \& Flegal, K.M. (2016). Tendencias en la prevalencia de la obesidad entre niños y adolescentes en los Estados Unidos, 1988-1994 a 2013-2014. Jama, 315(21), 2292-2299.

Panahi, S., \& Tremblay,A. (2018). Sedentariness and Health: is sedentary behavior more than just physical inactivity?. Front Public Health, 6, 1-7. https://doi.org/10.3389/ fpubh. 2018.00258

Parra, S. R., Gómez, M. G., \& Pintor, M. M. (2014). Factores que inciden en la implementación de las TIC en los pro- 
cesos de enseñanza-aprendizaje en $5^{\circ}$ de Primaria en Colombia. Revista Complutense de Educación, 26, 197-213. h t t p s : / / d o i . org/ $10.5209 /$ rev_RCED.2015.v26.46483

Peñarrubia-Lozano, C., Romero-Roso, L., Olóriz-Nivela, M., \& Lizalde-Gil, M. (2021). Challenge as a strategy to promote physical activity in university students. RETOS: Nuevas tendencias en educación física, deporte y recreación, 39, 58-64. https://doi.org/10.47197/retos.v0i39.78228

Práxades, A., Sevil, J., Moreno, A., del Villar, F., \& GarcíaGonzález, L. (2016). Niveles de actividad física en estudiantes universitarios: diferencias en función del género, la edad y los estados de cambio. Revista Iberoamericana de Psicología del Ejercicio y el Deporte, 11(1), 123-132.

Quevedo-Blasco, V. J., Quevedo-Blasco, R., \& Bermúdez, M. P. (2009). Análisis de la motivación en la práctica de actividades físico-deportivas en adolescentes. Revista de Investigación en Educación, 6, 33-42.

Rhodes, R. E., Janssen, I., Bredin, S. S., Warburton, D. E., \& Bauman, A. (2017). Physical activity: Health impact, prevalence, correlates and interventions. Psychology \& Health, 32(8), 942-975. https://doi.org/10.1080/ 08870446.2017 .1325486

Rico-Díaz, J., Arce-Fernández, C., Padrón-Cabo, A., PeixotoPino, L., \& Abelairas-Gómez, C. (2019). Motivaciones y hábitos de actividad física en alumnos universitarios (Motivations and physical activity habits in university students). RETOS: Nuevas tendencias en educación física, deporte y recreación, 36, 446-453. https://doi.org/ $10.47197 /$ retos.v36i36.69906

Rippe, J. M. (2019). Lifestyle Medicine 2019: deeper, broader, and more precise. American. Journal of Lifestyle medicine, 13(5), 436-439. https://doi.org/10.1177/ 1559827619845342

Rivera-Tapia, J. A., Cedillo-Ramírez, L., Pérez-Nava, J., Flores-Chico, B., \& Aguilar-Enriquez, R. I. (2018). Uso de tecnologías, sedentarismo y actividad física en estudiantes universitarios. Revista Iberoamericana de Ciencias, 5(1).

Sarmento, H., Clemente, F. M., Araújo, D., Davids, K., McRobert, A., \& Figueiredo, A. (2018). What performance analysts need to know about research trends in association football (2012-2016): A systematic review. J. Sports Med, 48, 799-836. https://doi.org/10.1007/ s40279-017-0836-6

Schinke, R. J., McGannon, K., \& Smith, B. (Eds.). (2016). Community based research in sport, exercise and health science. Routledge.

Schoeppe, S., Alley, S., Van Lippevelde,W., Bray, N.A., Williams, S. L., Duncan, M. J., \& Vandelanotte, C. (2016). Efficacy of interventions that use apps to improve diet, physical activity and sedentary behaviour: a systematic review. International Journal of Behavioral Nutrition and Physical Activity, 13(1), 127. https://doi.org/10.1186/s12966016-0454-y
Simons, M., Chinapaw, M. J. M., Bovenkamp, M., R de Boer, M., Seidell, J. C., Brug, J., \& deVet, E. (2014). Active video games as a tool to prevent excessive weight gain in adolescents: rationale, desing and methods of a randomized controlled trial. BMC Public Health, 14(1), 1-13. https: / / doi.org/10.1186/1471-2458-14-275

Sobko, T., \& Brown, G. (2019). Reflecting on personal data in a health course: integrating wearable technology and ePortafolio for eHealth. Australasian Journal of Educational Technology, 35(3), 55-70.

Spanakis, E. G., Santana, S., Tsiknakis, M., Marias, K., Sakkalis, V., Teixeira, A., Janssen, J. H., ... \& Tziraki, C. (2016). Technology-Based Innovations to Foster Personalized Healthy Lifestyles and Well-Being: A Targeted Review. Journal of medical Internet research, 18(6), e128. https: / / doi.org/10.2196/jmir.4863

Sullivan, A., \& Lachman, M. (2017). Behavior Change with Fitness Technology in Sedentary Adults: A Review of the Evidence for Increasing Physical Activity. Front Public Health, 4. https: / /doi.org/10.3389/fpubh.2016.00289 Tong, H., Coira, E., Tong., W., Wang.Y., Quiroz, J. C., . . .Laranjo, L. (2019). Efficacy of a mobile social networking intervention in promoting physical activity: quasi-experimental study. JMIR Mhealth Uhealth, 7(3), 1-15. https: / / doi.org/10.2196/12181

Vázquez, C. C., \& Mesa, M. D. C. C. (2012). Motivación de los estudiantes de la Facultad de Ciencias de la Educación (Universidad de Sevilla) hacia la práctica de actividad físico-deportiva. RETOS. Nuevas tendencias en educación física, deporte y recreación, 22, 57-61.

Vázquez, F. J. (2021). Una propuesta para gamificar paso a paso sin olvidar el currículum: modelo Edu-Game (A proposal to gamify step by step without forgetting the curriculum: Edu-Game model). RETOS: Nuevas tendencias en educación física, deporte y recreación, 39, 811-819. https: / / doi.org/10.47197/retos.v0i39.76808

Vidarte, J.A., Velez, C., Sandoval, C., \& Alfonso, M. L. (2011). Actividad física: estrategia de promoción de la salud. Hacia la Promoción de la salud, 16(1), 202-218.

Walsh, J. C., Corbett,T., Hogan, M., Duggan, J., \& McNamara, A. (2016). An mhealth intervention using a smartphone app to increase walking behavior in young adults: A pilot study. JMIR MHealth and UHealth, 4(3). https: / / doi.org / 10.2196/mhealth. 5227

Yun, L., Vanderloo, L., Berry, T. R., Latimer-Cheung, A. E., O’Reilly, N., Rhodes R. E., ... \& Faulkner, G. (2018). Assessing the social climate of physical (in)activity in Canada. BMC Public Health, 18(1), 1301. https: / / doi.org/ 10.1186/s12889-018-6166-2 